\title{
Crystal Structure of Indomethacin Polymorph $\delta$ Solved by 3D Electron Diffraction
}

\author{
Molly Lightowler ${ }^{1}$, Shuting $\mathrm{Li}^{2}$, Xiao $\mathrm{Ou}^{2}$, Xiaodong Zou${ }^{1}$, Ming $\mathrm{Lu}^{2}$, Hongyi $\mathrm{Xu}^{1}$ \\ ${ }^{1}$ Department of Materials and Environmental Chemistry, Stockholm University, Stockholm SE-106 91, Sweden, \\ ${ }^{2}$ School of Pharmaceutical Sciences, Sun Yat-sen University, Guangzhou 510006, China \\ molly.lightowler@mmk.su.se
}

\begin{abstract}
A 47-year-old polymorphic structure (Form $\delta$ ) of the drug indomethacin (IDM) has been solved by 3D electron diffraction (3D ED). Since its discovery in 1974, the structure of $\delta$-IDM had remained a mystery. By performing a unique crystallisation technique, we successfully cultivated its single crystal. The very thin, ribbon-like crystal was too small $(\sim 1 \mu \mathrm{m}$ in width) to be studied by X-ray diffraction -including even the third generation of synchrotron radiation. With the aid of 3D ED, we finally elucidated the crystal structure of $\delta$-IDM. The structure exhibits a very long $b$-axis with the slowest growth and shortest crystal dimension occurring along this direction. Consequently, reflections along $0 k 0$ were missing in the $3 \mathrm{D}$ ED data and the structure could not be solved by direct methods. Instead, simulated annealing was employed to overcome this problem. This work highlights the powerfulness of 3D ED for structure determination of small crystals, which complements X-ray diffraction.
\end{abstract}

Keywords: polymorphism, electron diffraction, crystallization 